\title{
BlancoSon: NUEVA VARIEDAD DE GARBANZO BLANCO (Cicer arietinum L.) PARA MÉXICO
}

\section{BlancoSon: A NEW VARIETY OF WHITE CHICKPEA (Cicer arietinum L.) FOR MEXICO}

\section{Pedro F. Ortega-Murrieta ${ }^{*}$, Gustavo A. Fierros-Leyva', Isidoro Padilla-Valenzuela ${ }^{2}$, Jorge A. Acosta-Gallegos ${ }^{3}$, Víctor Valenzuela-Herrera ${ }^{4}$, Erasmo Gutiérres-Pérez ${ }^{5}$, Franklin G. Rodríguez-Cota ${ }^{6}$, Brenda Z. Guerrero-Aguilar ${ }^{3}$ y Sixto Velarde-Félix ${ }^{4}$}

\begin{abstract}
'Instituto Nacional de Investigaciones Forestales, Agrícolas y Pecuarias (INIFAP), Campo Experimental Costa de Hermosillo, Hermosillo Sonora, México. ${ }^{2}$ NNIFAP, Campo Experimental Norman E. Borlaug, Cd. Obregón, Sonora, México. ${ }^{3}$ INIFAP, Campo Experimental Bajío, Celaya, Guanajuato, México. ${ }^{4}$ INIFAP, Campo Experimental Valle de Culiacán, Culiacán, Sinaloa, México. ${ }^{5}$ INIFAP, Campo Experimental Todos Santos, La Paz, Baja California Sur, México. ${ }^{\circ}$ INIFAP, Campo Experimental Valle del Fuerte, Juan José Ríos, Sinaloa, México.
\end{abstract}

*Autor de correspondencia (ortega.pedro@inifap.gob.mx; ortegampedro@gmail.com)

A nivel mundial existen varios problemas que limitan la producción del garbanzo, entre ellos se encuentran las enfermedades radiculares, las cuales son causadas por un complejo de hongos nativos del suelo, que pueden atacar el cuello o raíces de la planta en cualquier etapa de desarrollo. En las regiones Noroeste y Bajío de México se reportan estas enfermedades de la raíz, causadas principalmente por hongos del género Fusarium (FierrosLeyva et al., 2019).

En México, la región del Noroeste es la principal área productora de garbanzo blanco, lo cual ha sido posible por el uso de variedades mejoradas con tolerancia a los patógenos causantes de la "rabia" (Fusarium oxysporum f. sp. ciceris y F. solani). Con base en resultados de seis años de evaluación de líneas avanzadas de garbanzo en 28 localidades en los estados de Sonora, Sinaloa, Baja California Sur y Guanajuato, se seleccionó y propuso para registro la variedad de garbanzo blanco BlancoSon, la cual se describe a continuación.

La variedad BlancoSon se originó de la cruza entre los progenitores MUTTH $\times$ Blanco Sinaloa-92. El progenitor MUTTH es una línea con resistencia a F. oxysporum f. sp. ciceris y F. solani, de hoja simple, identificada y seleccionada en la Costa de Hermosillo, Sonora; mientras que Blanco Sinaloa-92 es una variedad liberada por el INIFAP en el Valle de Culiacán, Sinaloa (Gómez-Garza, 1993), de hoja compuesta, grano grande de color blanco cremoso y de amplia adaptación a diversos ambientes. La cruza se realizó en el ciclo 2000-2001 en el Campo Experimental Costa de Hermosillo del INIFAP, y se identificó como IIGH.2001.2. Para la selección de la variedad, el manejo de la población segregante fue el siguiente: de $F_{1}$ a $F_{3}$ se utilizó el método genealógico y la cosecha se realizó en forma masal. En la generación $F_{4}$ se realizó la selección y cosecha por planta individual; finalmente, en las generaciones $F_{5}$ a $F_{8}$ la cosecha se efectuó en forma masal. La genealogía final fue: IIGH.2001.2.-M-M-M-2H-M-M-M-M.

La línea experimental en la generación $F_{8}$ se consideró como línea élite y se denominó HOGA 2001-2-2. Esta línea élite se sembró en terrenos infestados naturalmente con 1000 a 1200 unidades formadoras de colonia (UFC) con los hongos F. oxysporum f. sp. ciceris y F. solani en localidades de la Costa de Hermosillo (28 $45^{\circ} 06^{\prime \prime} \mathrm{N}, 111^{\circ} 27^{\prime} 37^{\prime \prime} \mathrm{O}$, $70 \mathrm{msnm}$ ). Para determinar el potencial de rendimiento, ésta fue evaluada en los ciclos de 2011 a 2016 en Ensayos Regionales Uniformes de Rendimiento en parcelas de 6 a $9 \mathrm{~m}^{2}$ y en parcelas de validación de 500 a $1000 \mathrm{~m}^{2}$ en 28 localidades de México. Los datos de rendimiento de origen y cribado (usando malla de $9 \mathrm{~mm}$ de diámetro) se presentan en el Cuadro 1.

En las evaluaciones, la variedad BlancoSon superó en rendimiento de grano a las variedades testigo, mostró mayor tolerancia a enfermedades de la raíz y presentó una calidad del grano apta para el mercado internacional. En general, las características fenotípicas de BlancoSon son similares a la variedad Blanco Sinaloa-92 (Gómez-Garza, 1993) y a Blanoro (Ortega et al., 2016). Con respecto a reacción a enfermedades de la raíz, y tomando como base la escala indicada por la UPOV (2005), que considera valores de 1 a 9, donde 9 es el valor máximo de tolerancia y 1 el valor mínimo e indica susceptibilidad, la variedad BlancoSon mostró tolerancia en nivel 7 a las infecciones de F. oxysporum f.sp. ciceris y F. solani, mientras que Blanoro y Blanco Sinaloa-92 presentaron niveles de tolerancia de 5 y 3 , respectivamente, en promedio de 12 evaluaciones del 2011 a 2016 en la Costa de Hermosillo, Sonora, México. La 

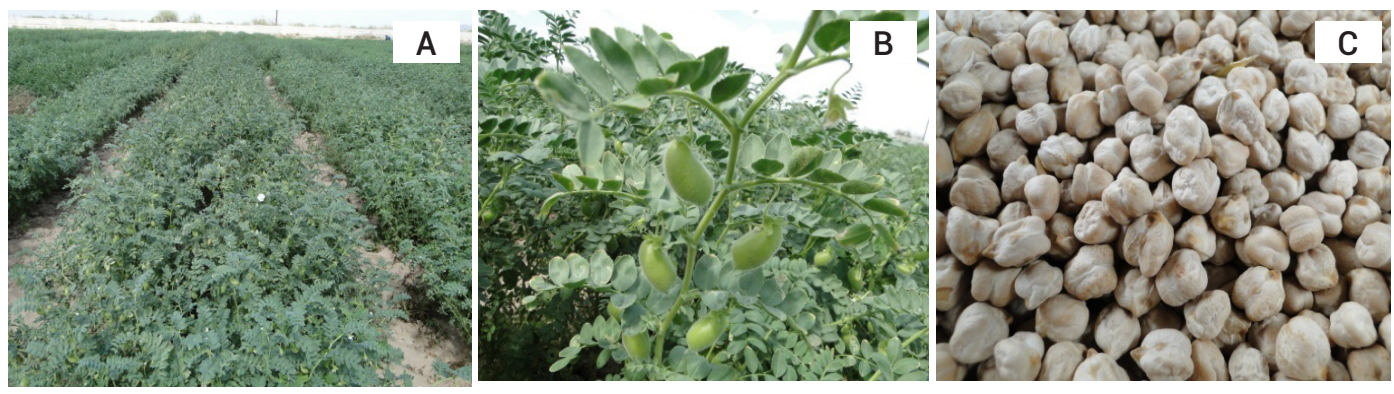

Figura 1. Porte de planta semierecta (A), hoja compuesta, tipo de vainas (B) y grano blanco cremoso y rugoso (C) de BlancoSon.

Cuadro 1. Rendimiento de origen y cribado $\left(\mathrm{kg} \mathrm{ha}^{-1}\right)$ de BlancoSon, Blanoro y Blanco Sinaloa-92, promedio de 28 localidades del Noroeste y Centro de México. Ciclos 2010-2011 y 2015-2016.

\begin{tabular}{lcccccc}
\hline Ciclo & \multicolumn{5}{c}{ Variedad/tipo de rendimiento } \\
\cline { 2 - 6 } Agrícola & \multicolumn{2}{c}{ BlancoSon } & \multicolumn{3}{c}{ Blanoro } & \multicolumn{2}{c}{ Blanco Sinaloa-92 } \\
\hline \multirow{2}{*}{ Origen } & Cribado & Origen & Cribado & Origen & Cribado \\
$2010-2011$ & 2971 & 2837 & 2922 & 2834 & 2776 & 2625 \\
$2012-2012$ & 3341 & 3119 & 3081 & 2908 & 2970 & 2694 \\
$2013-2014$ & 2772 & 2557 & 2478 & 2272 & 2188 & 1810 \\
$2014-2015$ & 2125 & 1913 & 1739 & 1474 & 1239 & 813 \\
$2015-2016$ & 1915 & 1596 & 1279 & 910 & 1816 & 1410 \\
Promedio & 2712 & 2436 & 1780 & 1573 & 1627 & 1339 \\
\hline
\end{tabular}

†Rendimiento cribado: después de pasar el grano por criba con perforaciones de 9 mm de diámetro.

variedad BlancoSon fue registrada en 2019, se le asignó el número GRZ-025-260319 en el Catálogo Nacional de Variedades Vegetales (CNVV) del Servicio Nacional de Inspección y Certificación de Semillas (SNICS) y el Título de Obtentor Número 2182 para el INIFAP.

Esta variedad es de ciclo intermedio (128 días en promedio a madurez fisiológica y 49 días a floración), altura de planta promedio de $59 \mathrm{~cm}$, con porte de planta semierecto y semiabierto, presenta en promedio tres ramas primarias y seis secundarias, las hojas son compuestas, con 17 foliolos de tamaño mediano, las flores de color blanco, las vainas de tamaño grande y la semilla es de color blanco cremoso, de tamaño grande (peso de 100 semillas de $64 \mathrm{~g}$ ), de forma angular, con rugosidad pronunciada, con una semilla por vaina (Figura 1).

Con base en el comportamiento de las pruebas realizadas en los ensayos regionales de rendimiento, la presente variedad se recomienda para su siembra en México en las regiones de la Costa de Hermosillo, los Valles del Yaqui y del Mayo en Sonora; Valle del Fuerte y Valle de Culiacán, en Sinaloa; Valle de Santo Domingo en Baja California Sur y región del Bajío en Guanajuato. La semilla original para la producción de semilla básica está resguardada en el Campo Experimental Costa de Hermosillo del INIFAP.

\section{AGRADECIMIENTOS}

Se agradecen los apoyos otorgados para la generación de la variedad a las siguientes instituciones y organizaciones: Fundación Pro Investigación Agrícola del Estado de Sonora A. C.-FIAES, Patronato para la Investigación y Experimentación Agrícola del Estado de Sonora A. C.-PIEAES, Asociación Agrícola Hermosillense S. A. de C. V., así como a las asociaciones de productores y a productores cooperantes de los estados de Sonora, Sinaloa, Baja California Sur y Guanajuato. 


\section{BIBLIOGRAFÍA}

Fierros-Leyva G. A., J. A. Acosta-Gallegos, P. F. Ortega-Murrieta, I. PadillaValenzuela, A. Álvarez-Bravo, M. Ramírez-Soto y S. Velarde-Félix (2019) Distribución de hongos asociados a pudriciones de la raíz del garbanzo. Revista Mexicana de Ciencias Agrícolas 10:131-142 https://doi.org/10.29312/remexca.v10i1.1730

Gómez-Garza R. M. (1993) Blanco Sinaloa-92: nueva variedad de garbanzo para el noroeste de México. Folleto Técnico Núm. 13. Campo Experimental Valle de Culiacán INIFAP. Culiacán Sinaloa, México.12 p.
Ortega M. P. F., G. A. Fierros L., I. Padilla V., V. Valenzuela H., J. A. AcostaGallegos, E. Gutiérres P., ... y F. G. Rodríguez C. (2016) Blanoro, nueva variedad de garbanzo blanco de grano extra grande para exportación. Revista Mexicana de Ciencias Agrícolas 7:209216 https://doi.org/10.13140/RG.2.1.4909.8647

UPOV Unión Internacional para la Protección de las Obtenciones Vegetales (2005) Garbanzo Código UPOV: CICER_ARI (Cicer arietinum) Directrices para la ejecución del examen de la distinción la homogeneidad y la estabilidad. Documento TG/143/4. Ginebra Suiza. 21 p. 
